\title{
The efficacy of continuous ropivacaine subfascial wound infusion after caesarean delivery in pain management: a prospective randomized controlled double-blind study.
}

\author{
Jerome Rosetti ${ }^{1}$, Jacques Francotte ${ }^{2}$, Emmanuel Noel ${ }^{2}$, Panagiotis Drakopoulos ${ }^{3}$, Nelson \\ Rabbachin $^{1}$, and Michael De Brucker ${ }^{2}$ \\ ${ }^{1} \mathrm{VUB}$ \\ ${ }^{2} \mathrm{CHU}$ Tivoli \\ ${ }^{3}$ UZ Brussel
}

May 5, 2020

\begin{abstract}
Objectives Analyze the efficacy in pain management of continuous ropivacaine subfascial wound infusion after caesarean delivery. Design Prospective, randomized controlled, double-blind study. Participants 69 caesarean section patients. Intervention After standardised spinal anesthesia (8-10 mg of 0.5\% hyperbaric bupivacaine combined with 2-2.5 $\mu \mathrm{g}$ of sufentanil) patients were randomly allocated: ropivacaine $0.2 \%$ infused through a subfascial wound catheter $(\mathrm{n}=35)$ or $\mathrm{NaCl} 0.9 \%$ $(\mathrm{n}=34)$, for 48 hours combined with recommended multimodal analgesia approach. Outcomes The primary outcome was the total amount of IV opioid use by patient-controlled analgesia in the first 48 hours after caesarean delivery. Secondary outcomes, assessed at regular intervals, were intensity of pain evaluated by VAS (0-10) at rest and at mobilisation, the incidence of post-operative nausea/vomiting and pruritus and time of first ambulation. Results Morphine consumption was significantly lower (mean \pm standard deviation), in the ropivacaine group (21.52 $\mathrm{mg} \pm 21.56)$ compared to the placebo group (29.57 mg $\pm 22.38 ; \mathrm{P}$ value $=0.047)$. No significant differences were observed in pain evaluated by VAS (mean \pm standard deviation), except for pain at mobilisation 6 hours after surgery (ropivacaine vs. placebo: $3.90 \pm 2.66$ vs. $5.36 \pm 2.55 ; \mathrm{P}$ value $=0.03$ ). No significant differences were observed in the incidence of post-operative nausea/vomiting and pruritus and time of first ambulation. Conclusion Continuous ropivacaine subfascial wound infusion can be considered as an effective analgesic method in addition to multimodal analgesia after caesarean delivery, resulting in less morphine consumption. Trail registration EudraCT 2017-004797-33 Funding none
\end{abstract}

\section{Introduction}

Caesarean section (CS) is one of the most common surgical procedures worldwide. In 2016, the rate of caesarean delivery was $18.6 \%$, ranging from $6 \%$ to $27.2 \%$ in the least and most developed countries, respectively. (1)

When obstetrical complications occur, CS is a life-saving procedure with clear maternal and foetal benefits. However, if there is no indication, it is not related to any maternal or foetal advantages.

As with any surgery, CS is associated with short- and long-term risks; principal among them are infectious, haemorrhagic, and thromboembolic. Postoperative pain management allows early rehabilitation and poor pain control results in decreased satisfaction with care, prolonged recovery time and increased use of health care resources and cost. (2)

Traditionally after major surgical procedures, opioids are used as first line post-operative medication for moderate to severe pain. Despite their efficacy, they are associated with unpleasant side effects, including 
nausea, vomiting, pruritus, drowsiness, and a risk for respiratory depression. In order to counter this, alternative pain killer techniques have emerged. Continuous subfascial wound infusion using a multi-orifice catheter with a local anaesthetic has been suggested as an effective method. However, data about its efficacy are lacking, given the conflicting results presented in randomized controlled trials. (3-13)

Since adequate postoperative pain management remains the primary goal after surgery, continuous wound infusion with a local anaesthetic could possibly improve patient's postoperative outcomes. The aim of this study was to evaluate the role in pain management of continuous ropivacaine subfascial wound infusion after caesarean delivery.

\section{Materials and methods}

\section{Study design}

The current study is a prospective randomized controlled double-blind study. For conduction of the study, institutional review board approval was obtained from the Ethical Committee of UZ Brussel (13/06/2018, reference number 2018/163) and the local Ethical Committee of Centre Hospitalier Universitaire Tivoli (05/04/2018, reference number 1245). This trial has been registered with EudraCT registry, number 2017004797-33, on the $17^{\text {th }}$ of March 2018.

\section{Study population}

A total of 80 patients treated by caesarean section between March 2018 and August 2019 at the Centre Hospitalier Universitaire Tivoli were initially selected. The first and last patient were respectively included on the $25^{\text {th }}$ of April 2018 and the $2^{\text {nd }}$ of August 2019. Elective as well as non-elective caesarean deliveries were included in this study. Other inclusion criteria were: gestation of more than 34 weeks, American Society of Anaesthesiology (ASA) I or II and [?] age 18 years. Exclusion criteria were allergies to analgesics, ASA III or higher, pre- or eclampsia, diabetes mellitus type I or II, postpartum haemorrhage (defined as [?] $1 \mathrm{~L}$ blood loss), BMI [?] 35, preoperative opioid consumption/abuse, psychiatric disorders, patient refusal to participate and language barrier. All patients signed an informed consent during prenatal consultation. Recruitment was performed by the gynaecologist in charge of the patient in the delivery room.

\section{Treatment}

To evaluate the effect of continuous ropivacaine subfascial wound infusion after caesarean delivery patients were randomly allocated in two groups receiving either ropivacaine $0.2 \%$ (Ropivacaine, $2 \mathrm{mg} / \mathrm{ml}$, Fresensius Kabi) (ropivacaine group) or $\mathrm{NaCl} 0.9 \%$ (placebo group) into the wound catheter. Group allocation was done using a computer-generated randomization and was concealed in sealed envelopes until start of surgery. Based on this information, a fix member of the midwife team who will otherwise not be involved in the study, prepared the injections for the elastomeric pump with either ropivacaine $0.2 \%$ or $\mathrm{NaCl} 0.9 \%$ so that only they were aware of the product that was administered through the catheter.

At the time of surgery, all of the patients received a standardized spinal anaesthesia with $8-10 \mathrm{mg}$ of hyperbaric bupivacaine $0.5 \%$ (Marcaine, AstraZeneca) combined with $2-2.5 \mu \mathrm{g}$ of sufentanil (Sufenta, Janssens-Cilag) based on clinicians' discretion. If inadequate spinal anaesthesia occurred, general anaesthesia was performed, and the patient had been excluded from the study.

All caesarean sections were done by Pfannenstiel incision followed by transverse lower uterine segment incision. Near the end of the procedure the parietal peritoneum was closed with a running absorbable suture. After this, a multi-holded catheter (PAINfusor catheter $15 \mathrm{~cm}$; Baxter, Amaro, Italy) was placed along the full length of the wound between the closed parietal peritoneum and the fascia transversalis. In the end, the gynaecologist closed the fascial layer and skin followed by securing the catheter to the skin. A 5 $\mathrm{ml}$ solution, ropivacaine $0.2 \%$ or $\mathrm{NaCl} 0.9 \%$, depending on group allocation, was administered through the catheter during the surgical intervention to evaluate his permeability. In the ropivacaine group, ropivacaine $0.2 \%$ was injected through the catheter, by an elastomeric pump infusor (Infusor $\mathrm{LV} 7 \mathrm{~mL} / \mathrm{h}$; Baxter) at 
a rate of $7 \mathrm{ml} / \mathrm{h}$ for 48 hours. In the placebo group, $\mathrm{NaCl} 0.9 \%$ was injected through the catheter, by an elastomeric pump infusor at a rate of $7 \mathrm{ml} / \mathrm{h}$ for 48 hours.

Post caesarean, all patients received a strictly identical multimodal analgesic treatment. A patient-controlled analgesia (PCA) device (CADD, Solis 2110, Smiths Medical, St. Paul, USA) was placed in the postanesthesia care unit (PACU) and set to deliver a $2 \mathrm{mg}$ dose of morphine with a 10-minute lockout time and a maximum allowed dose of $20 \mathrm{mg}$ per 4 hours. Paracetamol $1 \mathrm{~g}$ four times a day and diclofenac $75 \mathrm{mg}$ two times a day, both intravenous administrated, were systematically given in the first 48 hours. After 48 hours, the PCA device and all other intravenous therapies were stopped, and oral analgesics were given on demand based on the WHO ladder. Rescue intravenous antiemetics (metoclopramide $10 \mathrm{mg}$ and if insufficient ondansetron 4 $\mathrm{mg}$ ) were administered if postoperative nausea/vomiting developed. Pruritus was treated by subcutaneous naloxone $(0.4 \mathrm{mg}$ ) administration. Pre-operative ulcer prevention (ranitidine $150 \mathrm{mg}$ ) and post-operative thromboembolism prophylaxis (enoxaparine $0.4 \mathrm{ml}$ ) was performed according to international guidelines. Urine bladder catheter was left in place for at least 24 hours after surgery.

\section{Main outcome measures}

The primary outcome was the total amount of opioid use, measured on the PCA device, in the first 48 hours after caesarean delivery. Our secondary outcomes were intensity of pain, the incidence of adverse effects (post-operative nausea/vomiting and pruritus) and time of first ambulation. Pain was evaluated by visual analog scale (VAS) (0-10) at rest and at mobilisation (defined as pain during coughing) 2 hours, 6 hours and 12 hours after surgery (except if the patient was asleep) and thereafter once daily until discharge. The VAS tool consisted of a 10-cm horizontal line with at one end no pain and at the other end worst pain ever. Patients were asked to mark the point on the scale that corresponded to their pain. Post-operative nausea/vomiting and pruritus were evaluated using a categorical scale ranging from 0 to 2 ( 0 : none, 1 : mild (requiring no treatment) and 2: severe (requiring treatment)) at the same interval pain was assessed. Rescue medications for post-operative nausea/vomiting and pruritus were taken into account. All data were collected by independent investigators: nurses, midwifes, anaesthetists and gynaecologists that were not aware of group allocation.

\section{Statistical analysis}

Continuous variables were analysed using the independent t-test or Mann-Whitney U-test depending on the normality of the distribution. Normality was examined by the use of the Shapiro-Wilk test. Categorical variables were analysed by Pearson's chi-squared test or Fisher's exact test, as appropriate. All statistical tests used a two-tailed $\alpha$ of 0.05 . A P value $<0.05$ was considered statistically significant. Analyses were performed using STATA 13.0 (StataCorp. Stata Statistical Software: Release 13. CollegeStation, TX, USA).

\section{Sample size calculation}

The sample size calculation was based on the primary outcome of our study: the total amount of opioid use, measured on the PCA device, in the first 48 hours after surgery. Based on a mean post caesarean IV morphine consumption by PCA of $22 \mathrm{mg}$ (SD $8 \mathrm{mg}$ ), we calculated the simple sizes. (12) Using an alpha risk of 5 and a power of $95 \%$, each group required 34 patients to detect a reduction in morphine use of 30 $\%$.

\section{Results}

We initially selected 80 patients, of whom 11 were excluded after their caesarean section. Reasons for dropout were: four patients because of BMI [?] 35, three patients because they refused the prescribed medication doses after caesarean section, two patients because of ketamine administration during their caesarean section and two patients because of failed spinal analgesia. At the end, a total of 69 patients were included in the study and randomly allocated into two groups: 35 patients in the ropivacaine group, and 34 in the placebo group (Figure 1). Patient's baseline characteristics were comparable among the two groups (Table 1). As primary outcome, the morphine consumption (mean \pm standard deviation) was significantly reduced by 8.05 $\mathrm{mg}(\mathrm{P}$ value $=0.047)$ in the ropivacaine group compared to the placebo group, with respectively $21.52 \mathrm{mg}$ 
\pm 21.56 , and $29.57 \mathrm{mg} \pm 22.38$ morphine consumption (Table 2). For secondary outcomes no significant differences were observed in pain evaluated by VAS comparing the ropivacaine group to the placebo group, except for pain at mobilisation 6 hours after caesarean section. Pain by VAS at mobilisation 6 hours after surgery was $3.90 \pm 2.66$ in the ropivacaine group and $5.36 \pm 2.55$ in the placebo group (P value $=0.03$ ) (Figure 2). Pain by VAS at rest 6 hours after surgery (ropivacaine $2.73 \pm 1.98$ vs. placebo $3.79 \pm 2.43$ (P value $=0.08)$ ), at rest 12 hours after surgery (ropivacaine $3.53 \pm 2.19$ vs. placebo $3.67 \pm 2.46$ ( $\mathrm{P}$ value $=0.07)$ ) and at mobilisation 48 hours after surgery (ropivacaine $3.14 \pm 2.09$ vs. placebo $4.13 \pm 2.24(\mathrm{P}$ value $=0.06)$ ) showed a trend toward significance in favour of ropivacaine. No significant differences were observed for the incidence of adverse effects (post-operative nausea/vomiting and pruritus) (Figure 3). No significant differences were observed for time of first ambulation (Table 3). No pump failure or infection sites were observed. No breastfeeding problems were observed during hospitalisation.

\section{Discussion}

This study showed that continuous subfascial wound administration of ropivacaine combined to a multimodal systemic analgesia after caesarean section is more effective than placebo. Results showed a significant reduction in morphine consumption of $8.05 \mathrm{mg}$ in the ropivacaine group. On VAS, pain at mobilisation 6 hours after surgery was significantly reduced with ropivacaine compared to placebo. A trend toward significance in favour of ropivacaine was observed for pain by VAS at rest 6 hours after surgery, at rest 12 hours after surgery and at mobilisation 48 hours after surgery. In contrast, no significant differences were observed on VAS for pain 2 hours and 72 hours after surgery, both at rest and at mobilisation. For adverse effects (post-operative nausea/vomiting and pruritus) and time of first ambulation, no significant differences were observed comparing ropivacaine to placebo continuous subfascial wound infusion.

In obstetric populations, although several studies assessed analgesic effects of continuous anaesthetic wound infusion after caesarean section, clear results are still lacking because of conflicting results. (3-13) Furthermore, most of these studies showed some important limitations and heterogenicity in their designs, regarding the local anaesthetic agent used (ropivacaine, bupivacaine, levobupivacaine), its mode of release (continuous infusion or PCA), the NSAID in adjunction and the multimodal systemic analgesics used (morphine, oxycodone, ketoprofen or diclofenac) and their mode of release (oral, intramuscular intravenous or intrathecal).

A Cochrane Collaborative systematic review published in May 2010 concluded that morphine consumption was decreased by $1.70 \mathrm{mg}$ at 24 hours after caesarean section using anaesthetic wound infiltration compared to placebo. (7) This modest reduction compared to the $8.05 \mathrm{mg}$ reduction in our study may be explained by selection bias. Indeed Bamigboye et al. (2009) included next to wound infusions studies, studies of wound infiltration with local anaesthetic or with NSAID. (7) Furthermore, in all of the included wound infusion studies, the catheter was placed above the fascia. $(4,5,6)$ However, based on Rackelboom et al. (2010), better analgesia is obtained over 48 hours when ropivacaine and ketoprofen are infused below the fascia compared to administration above the fascia. (8) In this study, the multi-holded catheter was placed below the closed fascia transversalis and above the closed parietal peritoneum, to avoid intraperitoneal leakage.

In more recent studies, Kainu et al. (2012) found no benefit of continuous subfascial wound infusion with ropivacaine, which in their study failed to reduce the use of PCA administered oxycodone or pain scores compared to saline control. (10) The same conclusions were made in another randomized trial published by Reinikainen et al. (2014) where on the contrary ropivacaine was placed above the fascia and oxycodone not PCA administrated, possibly explaining the lack of efficacy from their ropivacaine infusion. (11) Conversely, Jolly et al. (2015) concluded that after caesarean section without subarachnoid morphine, continuous levobupivacaine compared to placebo subfascial wound infiltration decreased PCA administered morphine consumption $(6.7 \mathrm{mg}, \mathrm{P}$ value $=0.02)$ and pain intensity. (12) Nevertheless, the unblinded design of their study may have leaded to biases. Finally, Lalmand et al. (2017) investigated the duration and effect of intrathecal analgesia and continuous subfascial ropivacaine wound infiltration versus a control group after caesarean delivery. The duration of postoperative analgesia was increased with intrathecal morphine (380 minutes) and ropivacaine wound infusion (351 minutes) compared with the control (247 minutes), without significant difference between the morphine and catheter group. Cumulative postoperative morphine con- 
sumption was also significantly lower in the morphine group and catheter group compared to the control group. (13) When comparing continuous ropivacaine wound infusion after caesarean delivery to epidural morphine analgesia on pain evaluated by VAS, O'Neill et al. (2012) concluded a better analgesia, a lower incidence of side effects, less need for nursing care and shorter duration stay using wound infusion. (9) Based on the important role in post-operative pain of diffuse visceral nociceptive afferents in peritoneal tissue, blockade by local anaesthetics could easily explain the dose reduction of systemic analgesics.

Continuous subfascial wound infusion seems to be well tolerated by patients. In this study we selected ropivacaine as local anaesthetic because of its lower systemic toxicity and its shorter half-life comparing to bupivacaine and levobupivacaine. The infusion rate was chosen based on the study of Beaussier et al. showing that a constant rate of $0.2 \%$ ropivacaine at $10 \mathrm{~mL} / \mathrm{h}$ was well tolerated and associated with a sufficient margin of safety in colorectal surgery. (14) Liu's et al. meta-analysis concerning the use of continuous wound catheters delivering local anaesthetics reported a $1 \%$ technical pump failure rate and similar infection rates between active $(0.7 \%)$ and control groups (1.2\%). (15) Current study observed no technical failure and no infection issue, probably explained by the inadequate sample size for this purpose. The same concept can be applied to explain the fact that some of the secondary outcomes showed a trend in favour of the ropivacaine group without reaching the threshold of statistical significance: pain by VAS at rest 6 hours after surgery, at rest 12 hours after surgery and at mobilisation 48 hours after surgery. Nevertheless, a previous assessorblinded trial already evidenced beneficial effects of continuous subfascial wound infusion with ropivacaine after caesarean delivery, using pain at rest and at mobilisation on VAS as primary outcome. (9)

The strengths of the current study include the prospective, randomized controlled, double-blind design and the robust and rigorous methodological approach of the study protocol. Furthermore, using analgesic consumption on PCA device instead of VAS as primary outcome is likely to be more objective for pain evaluation. As far as we know, no other randomized controlled, double-blind studies were published comparing the analgesic efficacy of continuous ropivacaine versus placebo subfascial wound infusion after caesarean delivery using morphine consumption on PCA as primary outcome. The limitation of this study is the monocentric design.

In conclusion, continuous ropivacaine subfascial wound infusion trough a multi-holded catheter can be considered as an effective method for pain management in a multimodal analgesic approach after caesarean delivery and this without increasing the incidence of side effects.

\section{Conflict of interest}

None declared

\section{Contribution to authorship}

Jérôme Rosetti: conception of the study, planning and carrying out the protocol, data collection and management, writing and review of the article ; Jacques Francotte : conception of the study, planning and carrying out the protocol, review of the article; Emmanuel Noel : conception of the study, planning and carrying out the protocol, review of the article ; Panagiotis Drakopoulos : statistical work and review of the article, Nelson Rabbachin : data collection ; Michael de Brucker : conception of the study, planning and carrying out the protocol, data collection and management, writing and review of the article.

\section{Details of ethics approval}

Ethical Committee of UZ Brussel; date of approval: 02/05/2018; reference number: 2018/163. Ethical Committee of Centre Hospitalier Universitaire Tivoli; date of approval: 05/04/2018; reference number: 1245.

\section{Funding}

No external funding was used for this study.

\section{Acknowledgements}


The authors would like to thank all the gynaecologists, anaesthesiologists, midwifes and nurses of Centre Hospitalier Universitaire Tivoli for their devotion into this study.

\section{References}

1. Betrán AP, Ye J, Moller A-B, Zhang J, Gülmezoglu AM, Torloni MR. The Increasing Trend in Caesarean Section Rates: Global, Regional and National Estimates: 1990-2014. PloS One. 2016;11(2):e0148343.

2. Hoffmann B, Schorge J, Schaffer J, Halvorson L, Bradshaw K, Cunningham. Williams Gynecology. 2nd Edition. New York: McGraw-Hill Education; 2012.

3. Fredman B, Shapiro A, Zohar E, Feldman E, Shorer S, Rawal N, et al. The analgesic efficacy of patientcontrolled ropivacaine instillation after Cesarean delivery. Anesth Analg. 2000 Dec;91(6):1436-40.

4. Givens VA, Lipscomb GH, Meyer NL. A randomized trial of postoperative wound irrigation with local anesthetic for pain after cesarean delivery. Am J Obstet Gynecol. 2002 Jun;186(6):1188-91.

5. Zohar E, Shapiro A, Eidinov A, Fishman A, Fredman B. Postcesarean analgesia: the efficacy of bupivacaine wound instillation with and without supplemental diclofenac. J Clin Anesth. 2006 Sep;18(6):415-21.

6. Lavand'homme PM, Roelants F, Waterloos H, De Kock MF. Postoperative analgesic effects of continuous wound infiltration with diclofenac after elective cesarean delivery. Anesthesiology. 2007 Jun;106(6):1220-5.

7. Bamigboye AA, Hofmeyr GJ. Local anaesthetic wound infiltration and abdominal nerves block during caesarean section for postoperative pain relief. Cochrane Database Syst Rev. 2009 Jul 8;(3):CD006954.

8. Rackelboom T, Le Strat S, Silvera S, Schmitz T, Bassot A, Goffinet F, et al. Improving continuous wound infusion effectiveness for postoperative analgesia after cesarean delivery: a randomized controlled trial. Obstet Gynecol. 2010 Oct;116(4):893-900.

9. O'Neill P, Duarte F, Ribeiro I, Centeno MJ, Moreira J. Ropivacaine continuous wound infusion versus epidural morphine for postoperative analgesia after cesarean delivery: a randomized controlled trial. Anesth Analg. 2012 Jan;114(1):179-85.

10. Kainu JP, Sarvela J, Halonen P, Puro H, Toivonen HJ, Halmesmäki E, et al. Continuous wound infusion with ropivacaine fails to provide adequate analgesia after caesarean section. Int J Obstet Anesth. 2012 Apr;21(2):119-24.

11. Reinikainen M, Syväoja S, Hara K. Continuous wound infiltration with ropivacaine for analgesia after caesarean section: a randomised, placebo-controlled trial. Acta Anaesthesiol Scand. 2014 Sep;58(8):973-9.

12. Jolly C, Jathières F, Keïta H, Jaouen E, Guyot B, Torre A. Cesarean analgesia using levobupivacaine continuous wound infiltration: a randomized trial. Eur J Obstet Gynecol Reprod Biol. 2015;194:125-130.

13. Lalmand M, Wilwerth M, Fils J-F, Van der Linden P. Continuous Ropivacaine Subfascial Wound Infusion Compared With Intrathecal Morphine for Postcesarean Analgesia: A Prospective, Randomized Controlled, Double-Blind Study. Anesth Analg. 2017 Sep;125(3):907-12.

14. Beaussier M, El'Ayoubi H, Schiffer E, et al. Continuous preperitoneal infusion of ropivacaine provides effective analgesia and accelerates recovery after colorectal surgery: a randomized, double-blind, placebocontrolled study. Anesthesiology. 2007;107:461-468.

15. Liu SS, Richman JM, Thirlby RC, Wu CL. Efficacy of continuous wound catheters delivering local anesthetic for postoperative analgesia: a quantitative and qualitative systematic review of randomized controlled trials. J Am Coll Surg. 2006;203:914-932. 


\begin{tabular}{lcc} 
& Ropivacaine group & Placebo group \\
& $\mathbf{N}=\mathbf{3 5}$ & $\mathbf{N}=\mathbf{3 4}$ \\
\hline Age (years) & 32 & 34 \\
\hline Primiparous & $8(22.86 \%)$ & $11(32.35 \%)$ \\
\hline Multiparous & $27(77.14 \%)$ & $23(67.65 \%)$ \\
\hline BMl (kg/m ${ }^{2}$ ) & $29.64[ \pm 3.50]$ & $27.88[ \pm 3.99]$ \\
\hline Gestational age at delivery & 38 weeks and 3 days $[ \pm 1.81]$ & 38 weeks and 5 days $\pm[1.32]$ \\
\hline Cesarean indication & $9(25.71 \%)$ & $6(17.65 \%)$ \\
Urgent & $26(74.29 \%)$ & $28(82.35 \%)$ \\
\hline Elective & $3156.29[ \pm 571.06]$ & $3152.03[ \pm 585.30]$ \\
\hline Birthweight (gram) & $33(94.29 \%)$ & $31(91.18 \%)$ \\
\hline Breastfeeding & &
\end{tabular}

Table 1. Patient's baseline characteristics with age (years), gravity, BMI $\left(\mathrm{kg} / \mathrm{m}^{2}\right)$, gestational age at delivery (weeks and days), caesarean section indication, birthweight (gram) and number of breastfeeding patients. Data are presented as numbers (percentage) or as mean [ \pm standard deviation]

\begin{tabular}{|c|c|c|c|}
\hline & $\begin{array}{l}\text { Ropivacaine group } \\
\qquad \mathbf{N}=\mathbf{3 5}\end{array}$ & $\begin{array}{l}\text { Placebo group } \\
\qquad \mathbf{N}=\mathbf{3 4}\end{array}$ & P-value \\
\hline Morphine consumption & $21.52[ \pm 21.56]$ & $29.57[ \pm 22.38]$ & 0.047 \\
\hline
\end{tabular}

Table 2. Primary outcome corresponding to morphine consumption (mg) during the first 48 hours after caesarean delivery, measured on PCA device. Data are presented as mean [ \pm standard deviation].

\begin{tabular}{|c|c|c|c|}
\hline & $\begin{array}{l}\text { Ropivacaine group } \\
\qquad \mathrm{N}=\mathbf{3 5}\end{array}$ & $\begin{array}{l}\text { Placebo group } \\
\qquad N=\mathbf{3 4}\end{array}$ & P-value \\
\hline $\begin{array}{l}\text { Time of first ambulation } \\
\text { (minutes) }\end{array}$ & $1241[ \pm 5.34]$ & $1254[ \pm 5.84]$ & 0.9 \\
\hline
\end{tabular}

Table 3. Secondary outcomes corresponding to the time of first ambulation after caesarean section (CS). Data are presented as mean $[ \pm$ standard deviation].

\section{Hosted file}

Figure 1.docx available at https://authorea.com/users/298529/articles/427833-the-efficacyof-continuous-ropivacaine-subfascial-wound-infusion-after-caesarean-delivery-in-painmanagement-a-prospective-randomized-controlled-double-blind-study

\section{Hosted file}

Figure 2.docx available at https://authorea.com/users/298529/articles/427833-the-efficacyof-continuous-ropivacaine-subfascial-wound-infusion-after-caesarean-delivery-in-painmanagement-a-prospective-randomized-controlled-double-blind-study

\section{Hosted file}

Figure 3.docx available at https://authorea.com/users/298529/articles/427833-the-efficacyof-continuous-ropivacaine-subfascial-wound-infusion-after-caesarean-delivery-in-painmanagement-a-prospective-randomized-controlled-double-blind-study 